\title{
MAIT cells are licensed through granzyme exchange to kill bacterially sensitized targets
}

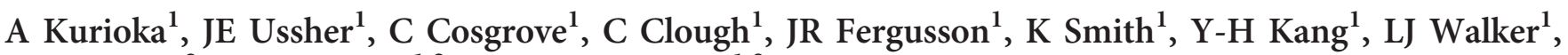 \\ TH Hansen ${ }^{2}, \mathrm{CB}$ Willberg ${ }^{1,3}$ and P Klenerman ${ }^{1,3}$
}

Mucosal-associated invariant T (MAIT) cells are an innate-like T-cell population restricted by the non-polymorphic, major histocompatibility complex class I-related protein 1, MR1. MAIT cells are activated by a broad range of bacteria through detection of riboflavin metabolites bound by MR1, but their direct cytolytic capacity upon recognition of cognate target cells remains unclear. We show that resting human MAITcells are uniquely characterized by a lack of granzyme (Gr) B and low perforin expression, key granule proteins required for efficient cytotoxic activity, but high levels of expression of GrA and GrK. Bacterial activation of MAIT cells rapidly induced GrB and perforin, licensing these cells to kill their cognate target cells. Using a novel flow cytometry-based killing assay, we show that licensed MAITcells, but not ex vivo MAITcells from the same donors, can efficiently kill Escherichia coli-exposed B-cell lines in an MR1- and degranulation-dependent manner. Finally, we show that MAIT cells are highly proliferative in response to antigenic and cytokine stimulation, maintaining high expression of GrB, perforin, and GrA, but reduced expression of GrK following antigenic proliferation. The tightly regulated cytolytic capacity of MAIT cells may have an important role in the control of intracellular bacterial infections, such as Mycobacterium tuberculosis.

\section{INTRODUCTION}

Mucosal-associated invariant T (MAIT) cells are an evolutionarily conserved sub-population of $\mathrm{T}$ cells in mammals, characterized by the expression of the semi-invariant $\mathrm{V} \alpha \mathrm{7.2}$-J $\alpha 33$ chain of the T-cell receptor (TCR), which restricts them to the nonpolymorphic, major histocompatibility complex class I-related protein 1, MR1 ${ }^{1-3}$ In murine models, MAIT cells are critical for the control of Bacillus Calmette-Guérin (BCG), ${ }^{4}$ Klebsiella pneumoni $a^{5}$ and Francisella tularensis ${ }^{6}$ infections. Their broad reactivity to microbes has recently been attributed to their ability to recognize ligands bound to MR1 originating from metabolic intermediates of riboflavin. ${ }^{7}$ MAIT cells are enriched in the intestine and liver, and represent $1-20 \%$ of CD $8^{+} \mathrm{T}$ cells in the periphery in man. ${ }^{1,8-10}$ In adult blood, MAIT cells are the dominant population among $\mathrm{CD} 8{ }^{+} \mathrm{T}$ cells that express the C-type lectin-like receptor CD161 at high levels. ${ }^{8,10,11}$ These $\mathrm{CD} 161^{++} \mathrm{CD} 8{ }^{+} \mathrm{T}$ cells are functionally and transcriptionally distinct from other $\mathrm{CD} 8^{+}$T-cell populations, ${ }^{11,12}$ with the ability to respond to cytokines interleukin-12 (IL-12) and IL-18 in a TCR-independent manner. ${ }^{13}$

Previous studies into CD161 ${ }^{++} /$MAIT cell effector functions have focused principally on their production of Tc1 and Tc17 cytokines. ${ }^{8,9,12-13}$ Recently, MAIT cells were shown to kill epithelial cells infected by Shigella dysenteriae. ${ }^{14}$ However, the factors regulating the cytotoxicity of MAIT cells and whether they can kill antigen-presenting cells (APCs), which in many infections provide a survival niche for intracellular bacteria, still remains to be addressed. This is a critical point, given their high frequencies in tissues, "effector-memory" phenotype ${ }^{11}$ and cytotoxic potential; such activity must be tightly regulated in order to limit immunopathology.

The primary mechanism by which cytotoxic cells induce apoptosis in tumor or virus-infected cells is granule exocytosis. This is dependent upon granzymes gaining entry to the cytoplasm of the target cell through the action of perforin; ${ }^{15}$ without perforin, internalized granzymes are retained in

\footnotetext{
${ }^{1}$ Peter Medawar Building for Pathogen Research, Nuffield Department of Medicine, University of Oxford, Oxford, UK. ${ }^{2}$ Department of Pathology and Immunology, Washington University School of Medicine, St Louis, Missouri, USA and ${ }^{3}$ National Institute for Health Research Oxford Biomedical Research Centre, University of Oxford, Oxford, UK. Correspondence: A Kurioka (ayako.kurioka@ndm.ox.ac.uk)
} 
endosome-like vesicles, and do not induce apoptosis. ${ }^{16}$ In humans, there are five granzymes with diverse substrate specificities: granzyme (Gr) A, GrB, GrH, GrK, and GrM. A hierarchy among these molecules determines cytotoxic efficiency, with perforin and GrB, but not GrA or GrK, correlating with lytic ability. ${ }^{17-20}$ Instead, recent studies have suggested that GrA and GrK are primarily non-cytotoxic molecules promoting proinflammatory responses. ${ }^{21-23}$ Perforin and GrB can be upregulated upon activation and proliferation, ${ }^{17,24}$ and are regulated by transcription factors such as T-bet and Blimp1..$^{25,26}$

In this study, we have performed a comprehensive characterization of the cytotoxicity of $\mathrm{CD} 161{ }^{++} \mathrm{CD} 8{ }^{+} \mathrm{T}$ cells, including the MAIT cells, and probe the relative contribution of TCR and innate cytokine signals regulating their granule content. Furthermore, we show that MAIT cells are highly proliferative in vitro and rapidly acquire high expression of GrB, GrA, and perforin. This cytotoxic phenotype "licenses" them to specifically kill target cells in an MR1-dependent manner.

\section{RESULTS}

\section{Resting blood-derived human MAIT cells have a unique cytotoxic profile}

First, we confirmed our previous finding ${ }^{12}$ that ex vivo, peripheral blood $\mathrm{CD} 161{ }^{++} \mathrm{CD} 8{ }^{+} \mathrm{T}$ cells expressed almost no GrB $(3.1 \pm 0.9 \%)$ compared with either $\mathrm{CD} 161^{+}$(47.6\%, $P<0.0001)$ or $\mathrm{CD} 161^{-}$sub-populations $(18.5 \%, P<0.01$; Figure 1a). The amount of perforin expressed by these cells, as measured by geometric mean fluorescence intensity, was also significantly lower than other $\mathrm{CD} 8{ }^{+}$T-cell sub-populations (both $P<0.0001$ ). In contrast, $\mathrm{CD} 161^{++} \mathrm{CD}^{+} \mathrm{T}$ cells expressed high levels of GrA $(96.6 \pm 1.2 \%)$ compared with $\mathrm{CD} 161^{+}$ $(87.2 \%, P<0.05)$ and $\mathrm{CD} 161^{-} \mathrm{CD}^{+}{ }^{+} \mathrm{T}$ cells $(31.3 \%, P<0.0001)$. $\mathrm{CD} 161^{++} \mathrm{CD} 8{ }^{+} \mathrm{T}$ cells also uniquely expressed high levels of GrK $(91.1 \pm 1.2 \%)$ compared with $\mathrm{CD} 161^{+} \mathrm{CD}^{+} \mathrm{T}$ cells $(32.9 \%, P<0.0001)$ and $\mathrm{CD} 161^{-} \mathrm{CD} 8^{+} \mathrm{T}$ cells $(16.3 \%$, $P<0.0001)$. Consistent with transcriptional profiling, ${ }^{11}$ cord blood $\mathrm{CD}_{161}{ }^{++} \mathrm{CD}^{+}{ }^{+} \mathrm{T}$ cells were also $\mathrm{GrA}^{+} \mathrm{GrK}^{+}$ (Supplementary Figure S1A online), suggesting this is an inherent feature of this subset.

Next, we asked whether this cytotoxic profile was characteristic of MAIT cells, or of the CD161 ${ }^{++} \mathrm{CD}^{+}$T-cell population as a whole. T cells possessing the V $\alpha 7.2$ TCR within the $\mathrm{CD} 161^{++} \mathrm{CD} 8{ }^{+} \mathrm{T}$-cell population are defined as MAIT cells throughout this study (Figure 1b). Within $\mathrm{CD} 161^{++}$ $\mathrm{CD}^{+}{ }^{+} \mathrm{T}$ cells, there was no difference in expression of $\mathrm{GrA}$ and perforin between $\mathrm{V} \alpha 7.2^{+}$cells and $\mathrm{V} \alpha 7.2^{-}$. However, GrB expression was significantly lower $(P<0.05)$ and GrK expression was significantly higher $(P<0.001)$ in the V $\alpha 7.2^{+}$MAIT cells (Figure 1c). Analysis of the simultaneous expression of perforin and granzymes in MAIT cells confirmed that the majority of the population coexpressed GrA and GrK, whereas a mean of $30 \%$ of MAIT cells coexpressed GrA, GrK, and perforin (Supplementary Figure S1B and C).

Using ImageStream technology, GrA was seen to colocalize with $\mathrm{CD} 107 \alpha$, a marker of cytotoxic granules, as well as with
GrK in $\mathrm{CD} 161^{++} \mathrm{CD} 8{ }^{+} \mathrm{T}$ cells (Figure 1d), suggesting that GrA and GrK are preformed within lytic granules. Thus, $\mathrm{CD} 161^{++} \mathrm{CD}^{+} \mathrm{T}$ cells, including the MAIT cells, have the potential to release $\mathrm{GrA}, \mathrm{GrK}$, and perforin immediately upon target cell interaction.

\section{Physiologic stimulation via MR1 leads to degranulation and changes in cytotoxic profile of MAIT cells}

We next investigated the regulation of cytotoxic potential in an in vitro model of MAIT cell activation. We have recently shown that MAIT cells can be activated both through the cognate interaction between MR1 and the TCR, as well as through IL-12 and IL-18 stimulation in a TCR-independent manner. ${ }^{13}$ Therefore, we tested if either pathway, or a combination of these signals, could induce a cytotoxic phenotype within MAIT cells.

Using our previously described model, ${ }^{13} \mathrm{THP} 1$ cell lines were pre-exposed to paraformaldehyde (PFA)-fixed Escherichia coli, and then cultured with enriched $\mathrm{CD} 8^{+} \mathrm{T}$ cells overnight, after which the cytotoxic profile of MAIT cells was evaluated (Figure 2). MAIT cells degranulated extensively in response to $E$. coli as measured by CD107 $\alpha$ expression (Figure 2a; 66.3\%, $P<0.0001$ ). This was almost completely blocked by an anti-MR1-blocking antibody (20.9\%; $P<0.0001$ ), whereas blocking IL-12 or IL-18 had minimal effect on the degranulation of the cells.

We then examined whether the expression of the key cytotoxic effector molecules, GrB and perforin, is modified by activation in this model. The frequency of MAIT cells expressing $\mathrm{GrB}$ increased to $\sim 35 \%$ in response to $E$. coli $(P<0.0001$; Figure 2b). Importantly, the addition of either anti-MR1-blocking antibody, or anti-IL-12-blocking antibody, significantly reduced the induction of $\mathrm{GrB}(6.5 \%, P<0.001$; $12.2 \%, P<0.001$, respectively), and the combination of antiMR1, anti-IL-12, and anti-IL-18-blocking antibodies inhibited GrB upregulation completely $(2.28 \%, P<0.001)$. Confirming the need for a TCR signal, direct stimulation of MAIT cells with anti-CD3/CD28/CD2 beads, but not with anti-CD3 beads or cytokines alone, also led to significant upregulation of $\mathrm{GrB}$ (Supplementary Figure S2A). This suggests that TCR signals are necessary for $\mathrm{GrB}$ upregulation in response to E. coli, and that costimulation either with $\mathrm{IL}-12$ or $\mathrm{CD} 2 / \mathrm{CD} 28$ is also required. Of note, there was a dose-dependent increase of $\mathrm{GrB}$ expression with increasing concentrations of E. coli (Supplementary Figure S3A and B). At the highest BpC, however, there was no further increase in $\mathrm{GrB}$ expression, although these cells were maximally activated as measured by CD69 expression (Supplementary Figure S3A). This may be due to the downregulation of the TCR upon exposure to high doses of bacteria, as shown by V $\alpha 7.2$ downregulation, in turn limiting further TCR-mediated upregulation of GrB. There was a loss of the CD161 ${ }^{++}$population with increasing doses of E. coli as previously described, ${ }^{27}$ but there was no visible loss of CD161 expression from the maximally activated MAIT cells (Supplementary Figure S3A). There was no difference in the frequency of MAIT cells or other $\mathrm{CD}^{+}{ }^{+}$T-cell populations when the cells were stained extracellularly or intracellularly for CD161 after activation (data not shown). Therefore, in this 

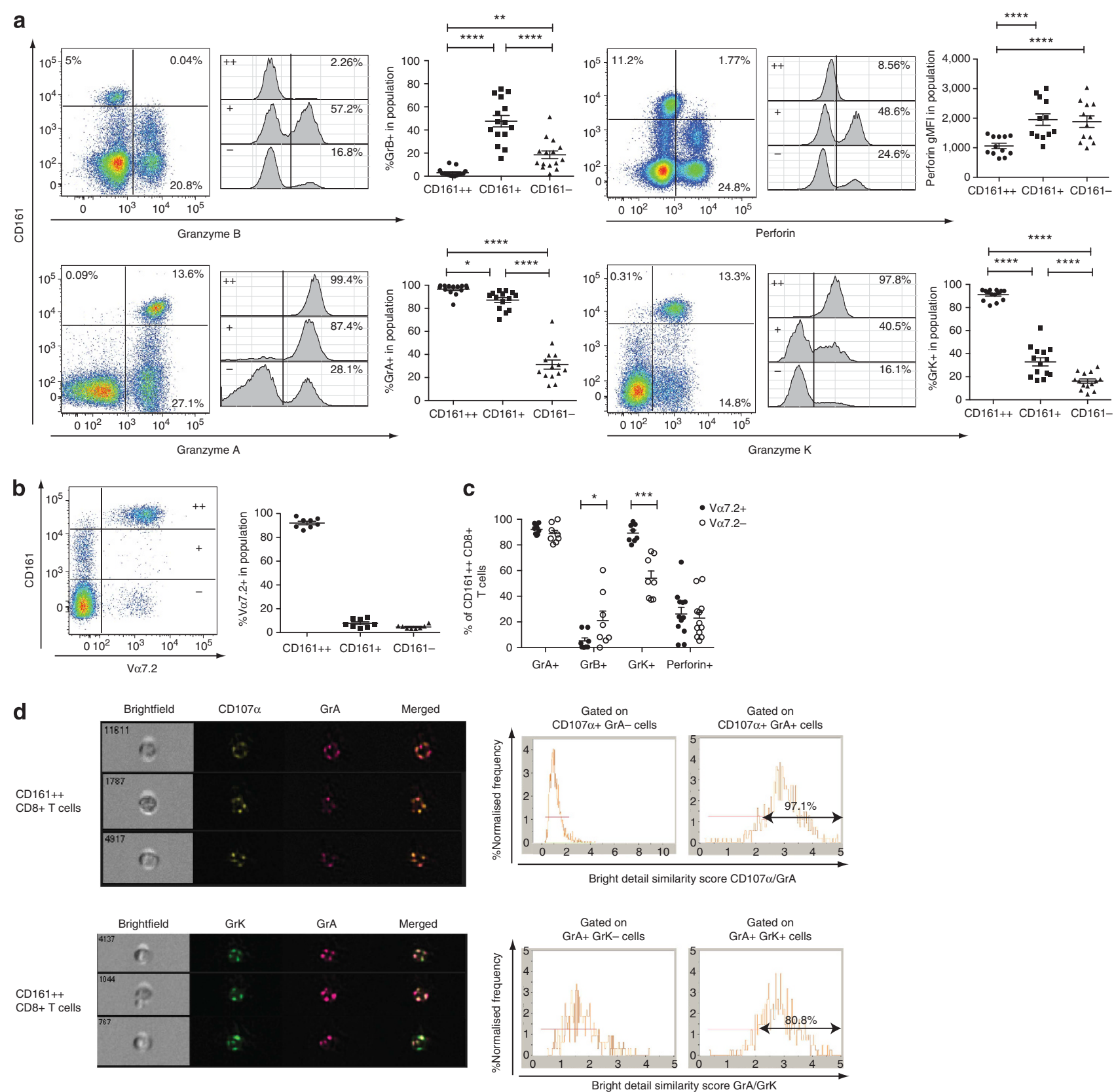

Figure 1 Resting blood-derived human mucosal-associated invariant T (MAIT) cells have a unique cytotoxic profile. (a) Expression of granzyme (Gr) B, perforin, $\mathrm{GrA}$, and $\mathrm{GrK}$ in peripheral blood $\mathrm{CD} 8^{+} \mathrm{T}$ cells from healthy donors according to CD161 expression levels. For each marker, representative staining gated on $\mathrm{CD} 8^{+} \mathrm{T}$ cells (left), representative staining gated on $\mathrm{CD} 161^{++}, \mathrm{CD} 161^{+}$, and CD161 ${ }^{-} \mathrm{CD} 8^{+} \mathrm{T}$ cells (middle), and cumulative data for 12-15 healthy individuals (right) are shown. Results shown as mean \pm s.e.m., analyzed by repeated-measures one-way analysis of variance (ANOVA), with Bonferroni's multiple comparisons test. ${ }^{*} P<0.05$, ${ }^{* \star} P<0.01,{ }^{* \star \star} P<0.001,{ }^{\star \star * \star} P<0.0001$. (b) CD $1611^{++} \mathrm{CD} 8^{+} \mathrm{T}$ cells are predominantly $\mathrm{V} \alpha 7.2^{+}$, compared with $\mathrm{CD}_{161}{ }^{+}$and CD161- $\mathrm{CD}^{+}{ }^{+} \mathrm{T}$ cells. Representative staining of $\mathrm{V} \alpha 7.2 \mathrm{~T}$-cell receptor (TCR) on CD8 ${ }^{+} \mathrm{T}_{\text {cells according to } \mathrm{CD} 161}$ expression levels and cumulative data are shown $(n=8)$. (c) Expression of GrB, perforin, GrA, and GrK in $V_{\alpha} 7.2^{+}$and $V_{\alpha} 7.2^{-}$cells, gated within the $\mathrm{CD}_{161}{ }^{++} \mathrm{CD}^{+} \mathrm{T}$-cell population. Cumulative data are shown, analyzed by paired $t$-test $(n=8-12)$. (d) Representative images showing Imagestream analysis of colocalization of GrA, GrK, and CD107 $\alpha$ expression within CD $1611^{++} \mathrm{CD}^{+} \mathrm{T}$ cells. Histograms of normalized cell frequency against colocalization as measured by Bright Detail Similarity scores; $>2.0$ is defined as colocalized (see Supplementary Methods).

activation model, we do not observe CD161 downregulation in MAIT cells.

We also observed perforin to be upregulated in this coculture model $(20.8 \%$ vs. $66.7 \%, P<0.0001$; Figure 2 c). However, unlike GrB, perforin upregulation could not be blocked by the addition of the MR1-blocking antibody. IL-12-blocking antibody also had only a small effect on the upregulation of perforin $(66.7 \%$ vs. $54.3 \%, P<0.01)$. Therefore, multiple factors may be contributing to perforin upregulation; indeed, direct stimulation of MAIT cells with IL-7, IL-15, or IL-2 upregulated perforin (Supplementary Figure S2B). This contrasts with the TCR- and costimulation-dependent GrB upregulation 
a

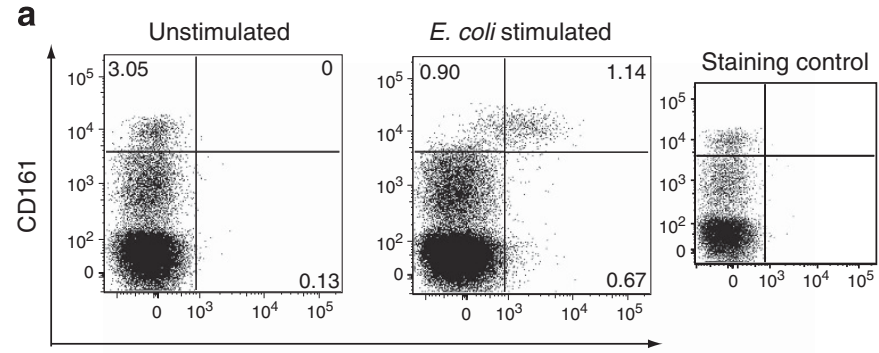

b $\quad \mathrm{CD} 107 \alpha$
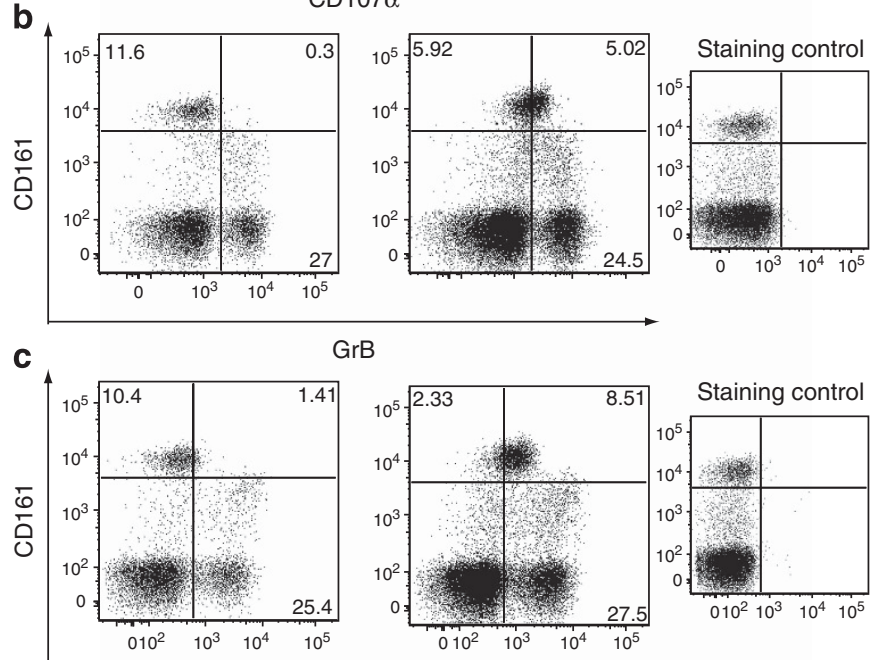

GrB
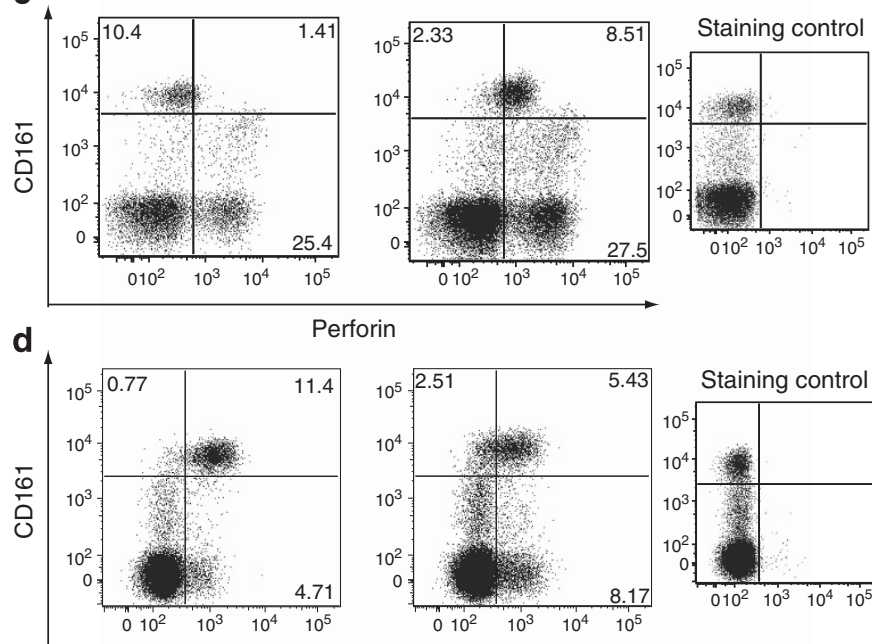

Perforin

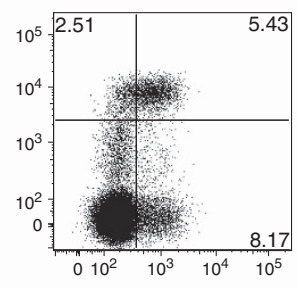

GrK

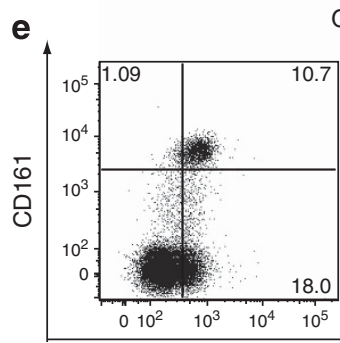

GrA
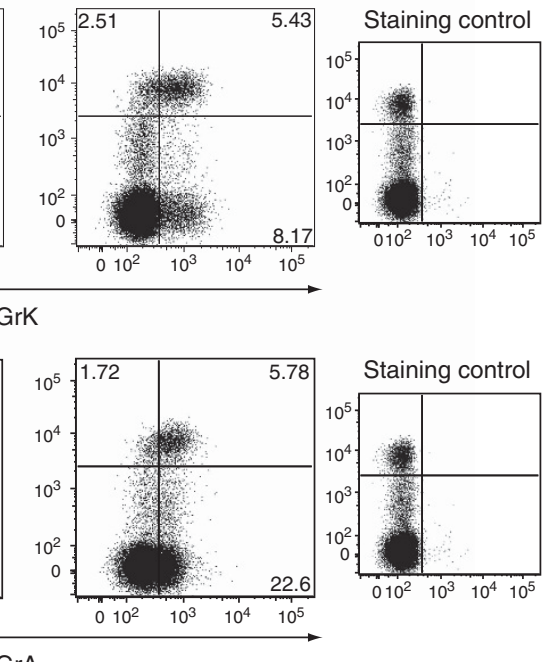

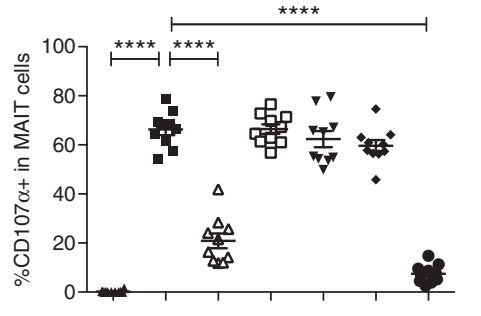

$\Delta$ Unstim

- Isotype

$\Delta$ Anti-MR1

口 Anti-IL-12

v Anti-IL-18

- Anti-IL-12/L-18

- Anti-MR1/LL-12/IL-18

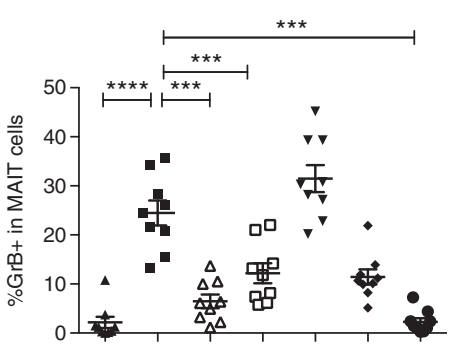

A Unstim

- Isotype

$\Delta$ Anti-MR1

口 Anti-IL-12

- Anti-IL-18

- Anti-IL-12/IL-18

- Anti-MR1/L-12/L-18
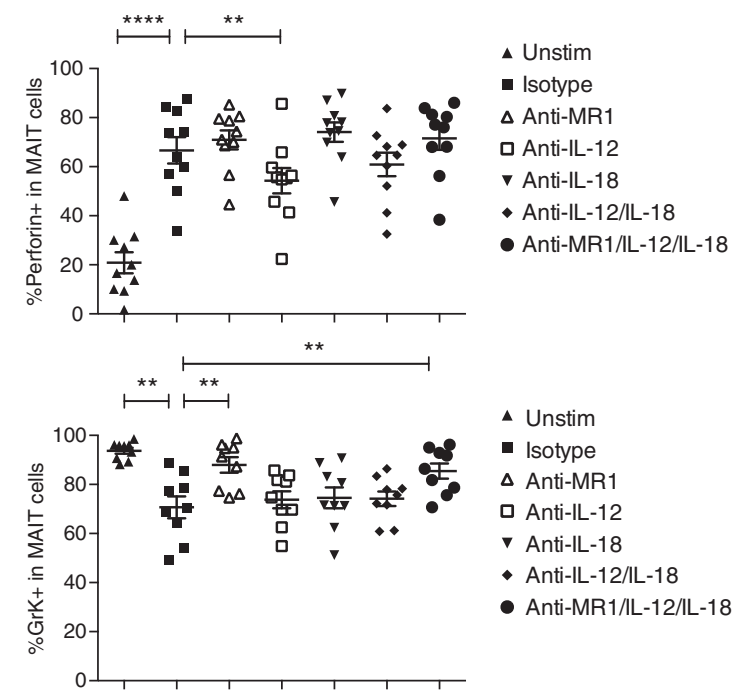

A Unstim

- Isotype

$\Delta$ Anti-MR1

a Anti-IL-12

v Anti-IL-18

- Anti-IL-12/IL-18

- Anti-MR1/LL-12/L-18

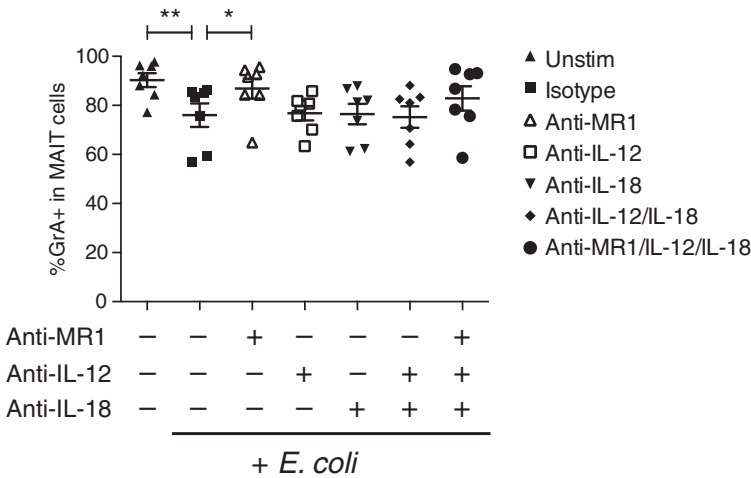

Figure 2 Bacterial stimulation leads to degranulation and changes in cytotoxic profile of mucosal-associated invariant T (MAIT) cells. Enriched CD8 ${ }^{+}$ T cells were cocultured with THP1 cells exposed to $E$. coli, in the presence or absence of blocking antibodies against MR1, interleukin (IL)-12, IL-18 or isotype controls, and assayed for the expression of (a) CD107 $\alpha$, (b) granzyme (Gr) B, (c) perforin, (d) GrK, and (e) GrA. Representative plots gated on CD8 ${ }^{+}$ $T$ cells are shown on the left panel and cumulative data on the right panel for each marker, with bars indicating mean \pm s.e.m. $(n=7-10)$. Staining control shows isotype control (conjugated with matched fluorochromes) used for gating for each marker. Note that GrA and GrK staining control is the same for each donor as both conjugated to the same flurochrome, and CD107 $\alpha$ staining uses the unstimulated control for gating. Results are analyzed by repeatedmeasures one-way analysis of variance (ANOVA), with Bonferroni's multiple comparisons test. ${ }^{\star} P<0.05,{ }^{\star \star} P<0.01,{ }^{\star \star \star \star} P<0.001,{ }^{\star \star \star \star \star} P<0.0001$.

described above, and suggests that GrB expression in MAIT cells may be more tightly regulated than perforin.

In contrast to GrB and perforin, the frequency of GrK- and GrA-expressing MAIT cells were both significantly reduced in response to E. coli (Figure 2d and e), and this loss was blocked by the anti-MR1 antibody. Stimulation of MAIT cells directly with anti-CD3/CD28/CD2 beads or phorbol 12-myristate 13 acetate/ ionomycin, but not cytokines, also reduced the percentage of 
MAIT cells expressing GrK, and to a limited extent, GrA, although this did not reach significance (Supplementary Figure S2C and D). There was also no significant increase in GrA or GrK expression as measured by geometric mean fluorescence intensity when cells were directly stimulated with cytokines, such as IL-12 + IL-18. Furthermore, there was no significant upregulation of granulysin or FasL when MAIT cells were stimulated with anti-CD3/CD28/CD2 beads or E. coli (Supplementary Figure S2E and F). Thus, MAIT cells modify their granule contents upon physiological activation.

\section{Licensed MAIT cells can kill target cells in an MR1- dependent manner}

MAIT cells are activated by a broad range of bacteria through recognition of their ligand, a metabolic precursor of riboflavin, presented by MR1. ${ }^{7}$ Whether this recognition leads to cytotoxicity, and what mechanisms are involved, have not been probed in detail. Furthermore, when administered to target cells, GrA and GrK, expressed by resting MAIT cells, have been suggested not to induce apoptosis, while GrB, not expressed by resting MAIT cells, induces apoptosis at equivalent concentrations. ${ }^{21,34}$

To test the capacity of ex vivo MAIT cells to kill target cells, a flow cytometry-based killing assay was developed, based on the published FATAL assay. ${ }^{28}$ Briefly, Epstein-Barr virustransformed B-cell lines (BCLs) were either incubated with PFA-fixed E. coli or sterility control overnight and stained with carboxyfluorescein succinimidyl ester (CFSE) and CellTrace Violet (CTV) dyes, respectively. These were mixed at a 1:1 ratio and cocultured with enriched CD8 ${ }^{+} \mathrm{T}$ cells at various E:T ratios. Specific killing of $\mathrm{CFSE}^{+}$target cells, but not $\mathrm{CTV}^{+}$ control cells, was then calculated based on the ratio of $\mathrm{CFSE}^{+}$ and $\mathrm{CTV}^{+}$cells in wells without effector cells. In addition, taking advantage of the capacity of modern flow cytometers to measure a greater number of parameters, $\mathrm{CD} 107 \alpha$ externalization by the $\mathrm{CD} 161^{++} \mathrm{CD} 8{ }^{+} \mathrm{T}$ cells was measured. Therefore, by combining the FATAL assay with the LAMP- 1 assay $^{29}$ and phenotyping the effector cells, our assay allows the identification of the cell population responsible for cytolysis; thus, removing the necessity to sort enrich specific or rare effector populations. The gating strategy is shown in Supplementary Figure S4A. Using this modified FATAL assay, we found that resting MAIT cells only killed $30 \%$ of E. coli-exposed BCLs even the highest E:T ratios, in an MR1-dependent manner (Figure $3 \mathbf{a}$ and $\mathbf{b}$ ).

Next, we asked whether pre-activation of MAIT cells, eliciting a more cytotoxic phenotype, would enhance their cytotoxic capacity. Peripheral blood mononuclear cells (PBMCs) isolated from the same donors were either pre-stimulated with PFAfixed $E$. coli for 6 days (licensed) or freshly thawed (ex vivo); both were enriched for $\mathrm{CD} 8^{+} \mathrm{T}$ cells in parallel. Licensed MAIT cells were significantly more cytotoxic compared with ex vivo MAIT cells, killing up to $70 \%$ of $E$. coli-exposed BCLs $(P<0.0001$; Figure $3 \mathbf{c}$ and $\mathbf{d})$. The frequency of $\mathrm{CD} 161{ }^{++} \mathrm{CD}^{+} \mathrm{T}$ cells did not significantly differ between ex vivo and licensed cells (Supplementary Figure S4B), and indeed post hoc adjustment of the E:T to the actual ratio of $\mathrm{CD} 161^{++} \mathrm{CD} 8{ }^{+} \mathrm{T}$ cells to target cells showed licensed MAIT cells were more cytotoxic on a per cell basis (Supplementary Figure S4C). Target cell killing by the E. coli-stimulated CD8 ${ }^{+}$ $\mathrm{T}$ cells was abolished with the addition of the anti-MR1blocking antibody, confirming MAIT cells were mediating the killing (Figure 3e). Inhibition of degranulation and perforin polymerization with ethylene glycol tetra-acetic acid also prevented killing, suggesting this process was degranulation mediated. Blocking FasL did not affect this killing, consistent with the low FasL expression on activated MAIT cells (Supplementary Figure S2F).

In addition, we confirmed that the $\mathrm{CD} 161^{++} \mathrm{CD} 8^{+}$T-cell population was responsible for target cell killing as only this $\mathrm{CD}^{+}{ }^{+}$T-cell population degranulated in response to E. coliexposed BCLs (Figure 3f and g). Furthermore, this novel cytotoxicity assay uses two amine-reactive dyes that bind to both cytosolic and membrane proteins, which are less prone to dye transfer than the lipophilic dye used in the original FATAL assay; thus, we were able to measure trogocytosis of the target cell membrane by the $\mathrm{T}$ cells, indicating prior synapse formation. ${ }^{30}$ This showed that $\mathrm{CD} 161^{++} \mathrm{CD} 8{ }^{+} \mathrm{T}$ cells made immunological synapses with the $\mathrm{CFSE}^{+}$target cells, and not with the $\mathrm{CTV}^{+}$control cells (Figure 3g and Supplementary Figure S3D). Ex vivo $\mathrm{CD} 161^{++} \mathrm{CD} 8{ }^{+} \mathrm{T}$ cells degranulated more readily than $E$. coli-stimulated $\mathrm{CD} 161{ }^{++} \mathrm{CD} 8{ }^{+} \mathrm{T}$ cells in response to the same target cells (Figure 3f), yet were less cytotoxic than the latter, consistent with the hypothesis that MAIT cells needed licensing by granzyme exchange to become highly cytotoxic. When $\mathrm{CD}_{107 \alpha^{+}}$cells within $\mathrm{CD} 161^{++} \mathrm{CD} 8{ }^{+} \mathrm{T}$ cells were compared, the frequency of cells that had acquired CFSE was significantly higher in the $E$. coli-stimulated cells than in ex vivo cells (Figure $3 \mathbf{h}$ ), suggesting there is also increased synapse formation between the target cells and E. coli-stimulated CD161 ${ }^{++} \mathrm{CD} 8{ }^{+} \mathrm{T}$ cells compared with ex vivo $\mathrm{CD} 161^{++} \mathrm{CD} 8{ }^{+} \mathrm{T}$ cells.

Overall, these data suggest that $E$. coli-stimulated, licensed MAIT cells maintain the capacity to degranulate and possess enhanced killing capacity compared with ex vivo MAIT cells.

\section{Rapid GrB upregulation in MAIT cells is associated with Blimp1 and T-bet expression}

Next, we probed the kinetics of this MAIT cell licensing by examining $\mathrm{GrB}$ and perforin upregulation in response to $E$. coli. MAIT cells showed no change in the expression of GrB and perforin within the first $6 \mathrm{~h}$ of stimulation (Figure $4 \mathrm{a}$ and $\mathbf{b}$ ), but markedly upregulated these effector molecules by $18 \mathrm{~h}$, with most cells expressing GrB and perforin by $30 \mathrm{~h}$. This upregulation was specific to the MAIT cell population, the kinetics of which was similar to anti-CD3/CD28/CD2 stimulation (Figure $4 \mathrm{c}$ and $\mathbf{d}$ ).

The transcription factors T-bet and Blimp1 are both important for $\mathrm{GrB}$ expression in $\mathrm{CD}^{+} \mathrm{T}$ cells. ${ }^{25,26}$ To investigate whether these transcription factors are associated with $\mathrm{GrB}$ upregulation in MAIT cells, PBMCs were stimulated with E. coli for $24 \mathrm{~h}$, with or without the addition of MR1- and IL-12- 


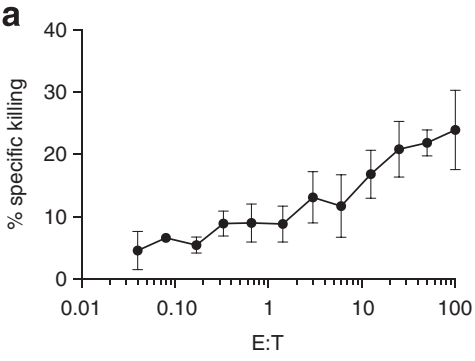

d

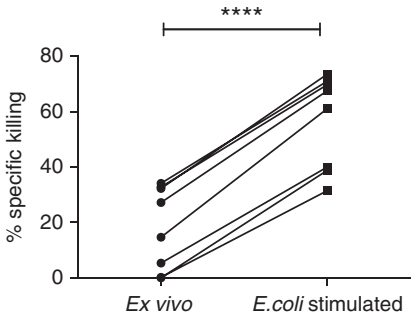

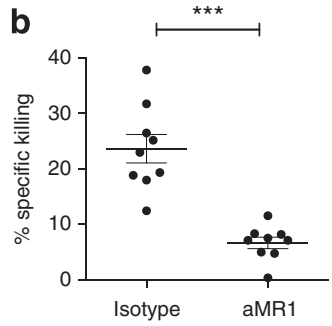

e

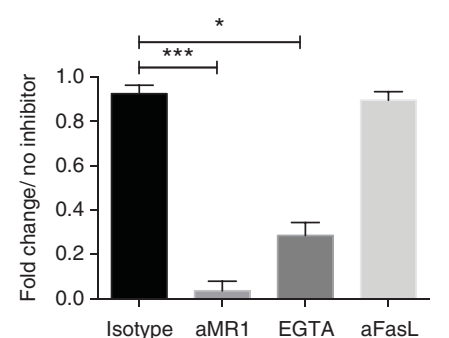

C

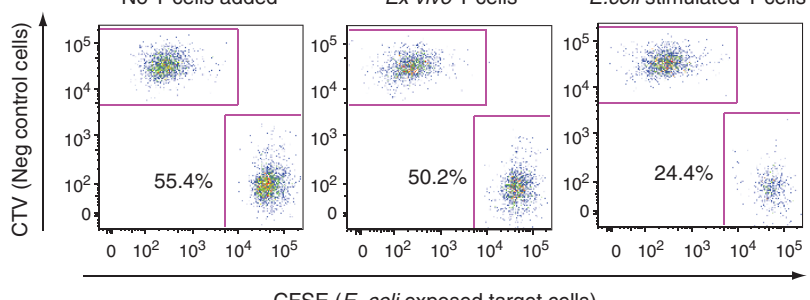

CFSE (E. coli exposed target cells)

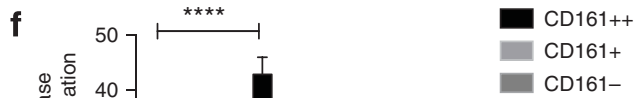

g

Gated on CD161++ CD8+ T cells
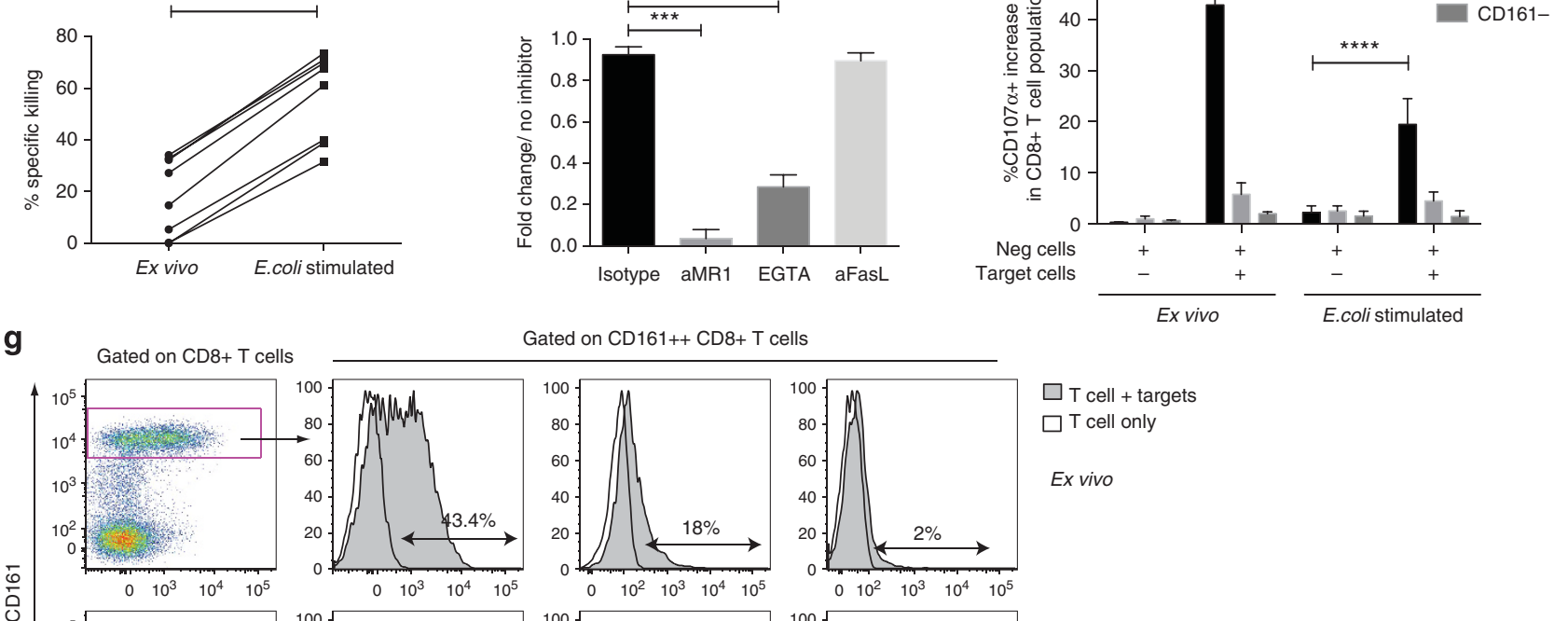

$\square$ T cell + targets $\square$ T cell only

Ex vivo
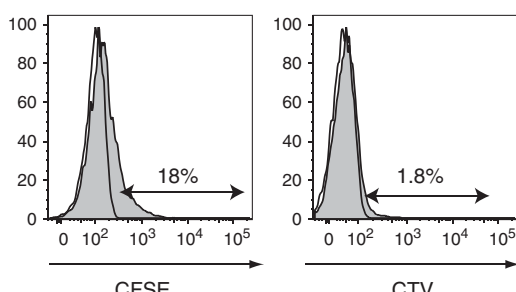

E. coli

stimulated

CTV

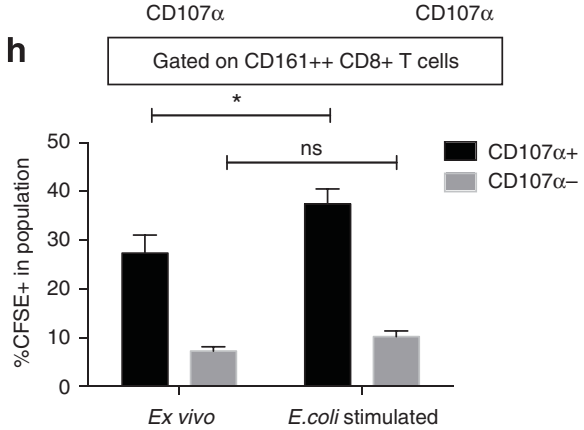

Figure 3 E.coli-stimulated mucosal-associated invariant T (MAIT) cells can efficiently kill target cells in an MR1-and degranulation-dependent manner. B-cell lines (BCLs) were either incubated with paraformaldehyde (PFA)-fixed $E$. coli or sterility control and stained with carboxyfluorescein succinimidyl ester (CFSE) and CellTrace Violet (CTV) dyes, respectively, and cocultured with enriched CD8 ${ }^{+} \mathrm{T}$ cells. (a) Percentage of specific killing of target BCLs by ex vivo MAIT cells at various E:T ratios. Mean \pm s.e.m. of duplicate results of three independent experiments shown $(n=10)$. (b) Percentage of specific killing of target cells by ex vivo MAIT cells with and without an anti-MR1-blocking antibody at E:T $=50: 1$. $(n=9)$. (c) Example plots showing the frequency of live E. coli-exposed BCLs and negative control BCLs without effector cells (left), with ex vivo MAIT cells (middle), or with MAIT cells stimulated with E. coli for 6 days (right), added at E:T=10:1. (d) Percentage of specific killing of target cells by ex vivo and E. coli-stimulated MAIT cells from the same donors at $\mathrm{E}: \mathrm{T}=10: 1$. Mean \pm s.e.m. of duplicate results of three independent experiments are shown. $(n=8)$. (e) Inhibition of killing at E:T=10:1 by E. coli-stimulated MAIT cells in presence of anti-MR1 antibody, ethylene glycol tetra-acetic acid (EGTA), or anti-FasL antibody, compared with killing with no inhibitor. Repeated-measures one-way analysis of variance (ANOVA), with Bonferroni's multiple comparisons test. ( $n=5)$ (f) Cumulative data showing increase in \%CD107 $\alpha^{+}$cells in indicated CD8 ${ }^{+}$T-cell populations compared with wells with T cells only. Analyzed by two-way ANOVA, comparing increase in CD107 $\alpha^{+}$expressing cells when target cells are added, within each $\mathrm{CD}^{+}{ }^{+} \mathrm{T}$-cell population, with Bonferroni's multiple comparisons test. CFSE ${ }^{+}$E. coli-exposed BCL $=$Target cells, $\mathrm{CTV}^{+}$negative control BCL $=$Neg cells. $(n=8)$. (g) Representative plots of ex vivo and E. coli-stimulated CD8 T cells from the killing assays. Histograms showing expression of CD107 $\alpha$, and CFSE and CTV acquired through trogocytosis in CD $161^{++} \mathrm{CD}^{+}$T cells. (h) Trogocytosis of CFSE from E. coli-exposed BCL membranes onto degranulating CD161 ${ }^{++} \mathrm{CD} 8^{+}$T cells $(n=8)$. Analyzed by two-way ANOVA, comparing wells with ex vivo and $E$. coli-stimulated cells, with Bonferroni's multiple comparisons test. NS, nonsignificant. ${ }^{*} P<0.05$, ${ }^{\star * \star} P<0.001,{ }^{* * \star *} P<0.0001$. 

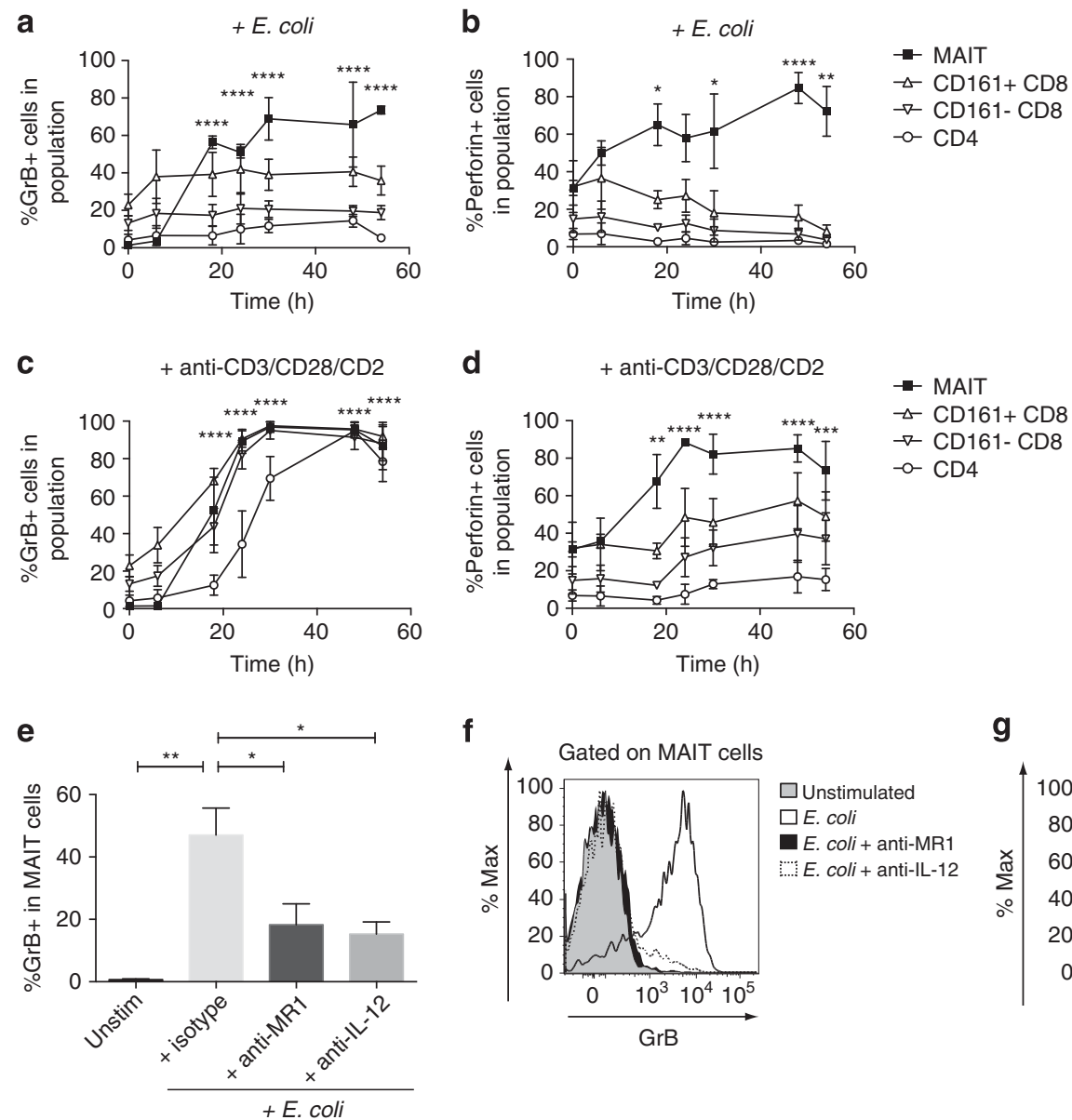

f

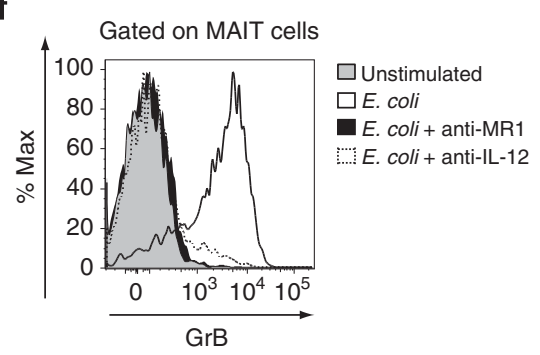

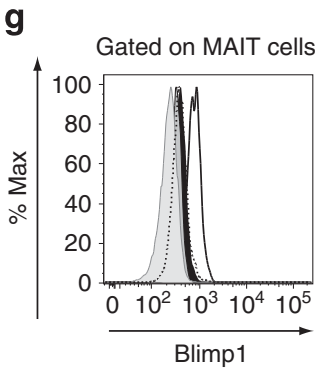

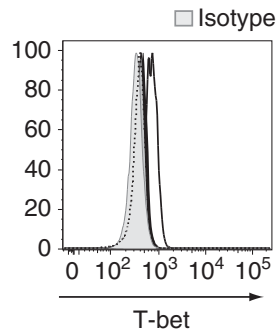

h

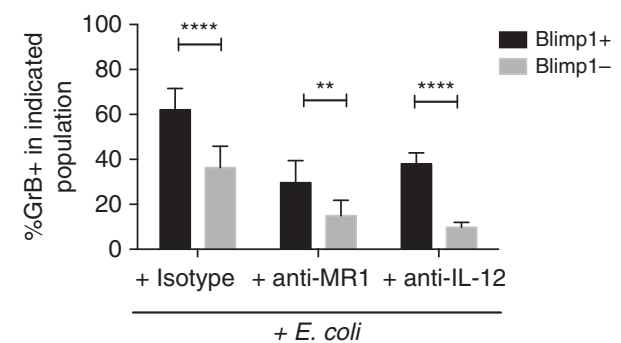

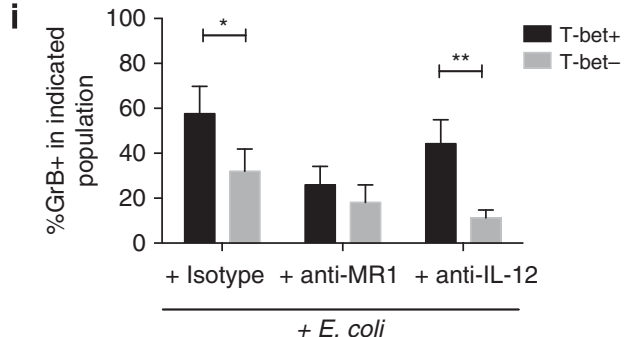

Figure 4 Granzyme (Gr) B and perforin upregulation in E. coli-stimulated mucosal-associated invariant T (MAIT) cells is rapid and associated with Blimp1 and T-bet expression. (a-d) Peripheral blood mononuclear cells (PBMCs) were stimulated with (a, b) paraformaldehyde (PFA)-fixed E. coli, or (c, d) anti-CD3/CD28/CD2 coated beads, and stained for GrB and perforin at the indicated time points $(n=3)$. Asterisks $\left({ }^{*}\right)$ indicate significant increases in effector molecules in MAIT cells compared with the unstimulated controls, by a two-way analysis of variance (ANOVA), with Bonferroni's multiple comparisons test. (e-i) PBMCs were stimulated with PFA-fixed $E$. colifor $24 \mathrm{~h}$ with and without blocking antibodies to MR1 and interleukin (IL)-12, and stained for GrB, T-bet, and Blimp1 $(n=7)$. (e) Cumulative data for frequency of GrB expressing MAIT cells and (f) representative example of GrB expression in MAIT cells after E. coli stimulation, \pm blocking antibodies to MR1 or IL-12. One-way ANOVA, Bonferroni's multiple comparisons test. (g) Representative T-bet and Blimp1 expression in MAIT cells unstimulated or stimulated with PFA-fixed E. coli \pm blocking antibodies to MR1 or IL-12. For figure legend, see panel f. (h) Frequency of GrB-expressing cells in T-bet ${ }^{+}$or T-bet $^{-}$MAIT cells. (i) Frequency of GrB-expressing cells in Blimp ${ }^{+}$or Blimp1 ${ }^{-}$MAIT cells. Results analyzed by two-way ANOVA with Bonferroni's multiple comparisons test. Bars indicate mean \pm s.e.m. ${ }^{\star} P<0.05,{ }^{* \star} P<0.01,{ }^{* \star \star} P<0.001,{ }^{* \star \star *} P<0.0001$.

blocking antibodies. As expected from the THP1 coculture model (Figure 2), MAIT cells stimulated with E. coli upregulated GrB (Figure 4e and $\mathbf{f}$ ), but also coexpressed T-bet and Blimp1 in an MR1- and IL-12-dependent manner (Figure 4g). Cells expressing T-bet or Blimp1 were more likely to express $\mathrm{GrB}$ (Figure $4 \mathbf{h}$ and $\mathbf{i}$ ), supporting a role for these transcription factors in the upregulation of GrB in MAIT cells.

\section{MAIT cells readily proliferate in response to $E$. coli and maintain a highly cytotoxic profile}

Finally, we asked whether the granule content of MAIT cells were sustained during proliferation. When CTV-labeled PBMCs were cultured with PFA-fixed E. coli for 6 days, MAIT cells proliferated in a dose-dependent manner to E. coli (Figure $5 \mathbf{a}$ and $\mathbf{b}$ ). The proliferation of MAIT cells was 

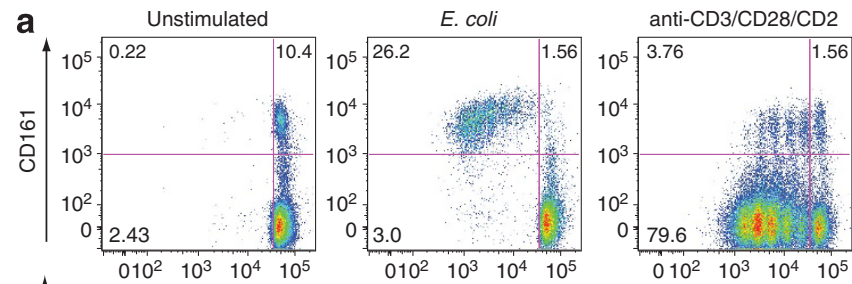

b
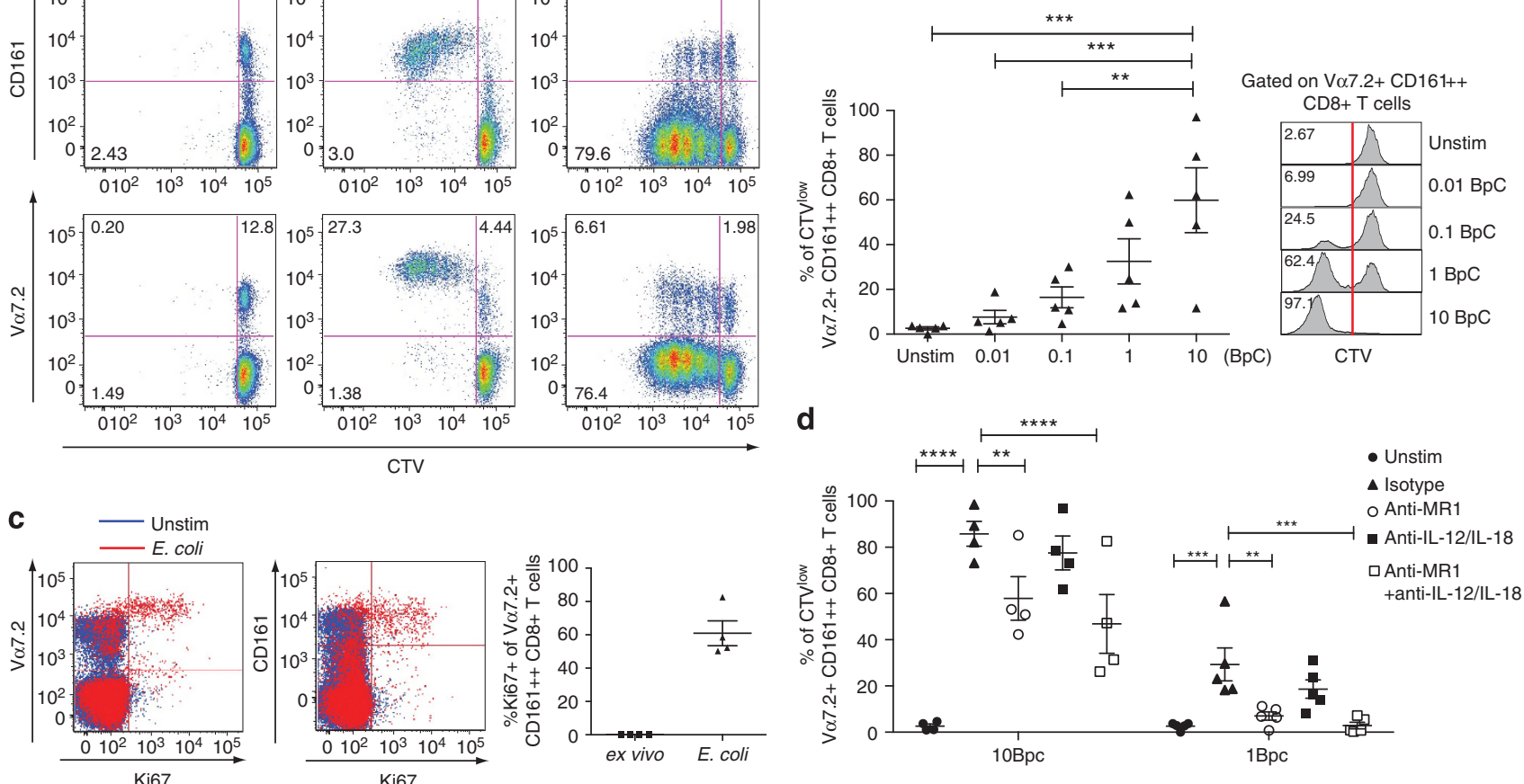

\section{d}

e

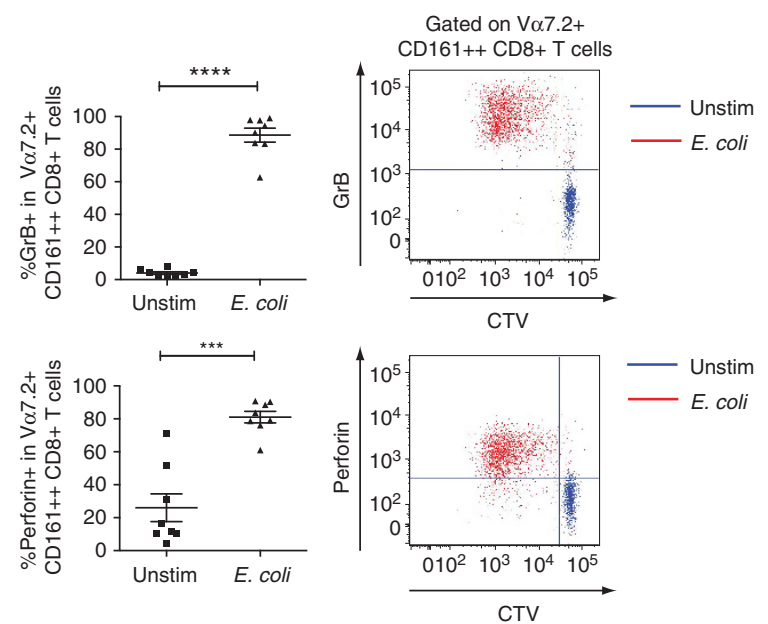

f

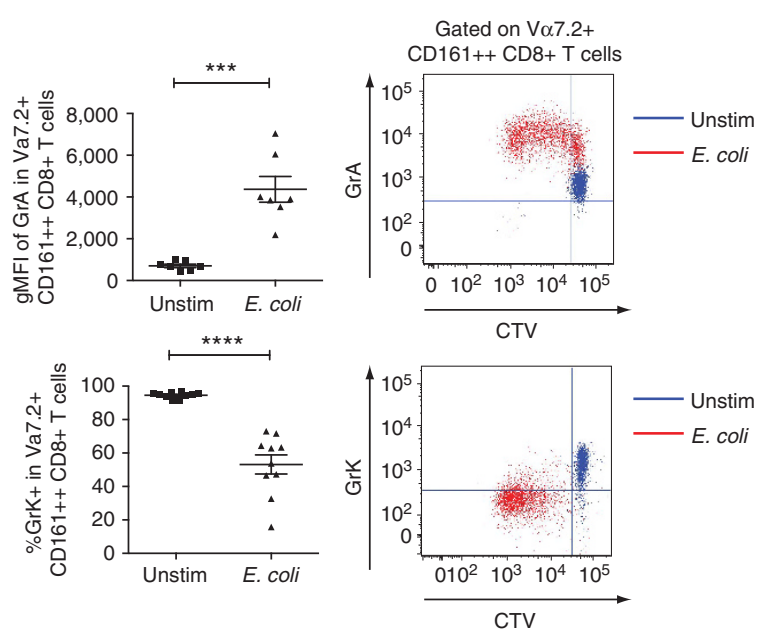

Figure 5 Mucosal-associated invariant T (MAIT) cells are readily proliferative in response to $E$. coli and maintain a highly cytotoxic profile. Peripheral blood mononuclear cells (PBMCs) from healthy donors were CellTrace Violet (CTV)-labeled and cultured with paraformaldehyde (PFA)-fixed E. colifor 6 days. $\mathrm{BpC}=$ bacteria per cell ratio. (a) Representative plot showing dilution of CTV against CD161 expression (upper panel) and V $\alpha 7.2$ expression (lower panel) at $10 \mathrm{BpC}$ or with anti-CD3/CD28/CD2 beads, gated on CD8 ${ }^{+} \mathrm{T}$ cells. (b) Cumulative data (left) and representative flow cytometry histograms are shown for each BpC. One-way analysis of variance (ANOVA), with Bonferroni's multiple comparisons test. (c) Representative plot (left) and cumulative data (right) showing Ki67 staining in $\mathrm{CD}^{+} \mathrm{T}$ cells after $E$. coli-stimulation at $10 \mathrm{BpC}(n=4)$. (d) Proliferation of $\mathrm{V} \alpha 7.2^{+} \mathrm{CD} 161^{++} \mathrm{CD} 8{ }^{+} \mathrm{T}$ cells in response to $E$. coli with anti-MR1, interleukin (IL)-12, and IL-18-blocking antibodies at the indicated BpCs. Results pooled from two independent experiments and analyzed by two-way ANOVA, with Bonferroni's multiple comparisons test. (e) GrB and perforin, and (f) GrA and GrK expression in CTVlabeled $\mathrm{V} \alpha 7.2^{+} \mathrm{CD} 161^{++} \mathrm{CD} 8{ }^{+} \mathrm{T}$ cells following E. colistimulation for 6 days. Cumulative data (left) and representative staining is shown (right). Gating for granzymes and perforin based on isotype controls. Results analyzed by paired $t$-test $(n=8)$. Bars indicate mean \pm s.e.m. ${ }^{\star} P<0.05$, ${ }^{\star \star} P<0.01$, ${ }^{\star \star \star *} P<0.001,{ }^{\star \star * \star} P<0.0001$.

confirmed by Ki67 staining (Figure 5c), and was inducible when enriched $\mathrm{CD}^{+} \mathrm{T}$ cells were directly stimulated with E. coli-exposed THP1 cells (Supplementary Figure S5A), confirming this is a specific effect and not bystander proliferation. Interestingly, although most cells remained CD161 ${ }^{++}$ (Figure 5a, Supplementary Figure S5A), CD161 was downregulated on MAIT cells with increasing rounds of cell divisions (Supplementary Figure S5B). In addition, although both $\operatorname{CD} 8 \alpha \alpha$ and $C D 8 \alpha \beta$ subsets of MAIT cells proliferated, there was greater proliferation of the $\operatorname{CD} 8 \alpha \beta$ subset (Supplementary Figure S5C). To investigate the relative contributions of the TCR signal and innate cytokines for MAIT 
cell proliferation, blocking antibodies to MR1 or IL-12 and IL18 were added. Addition of the MR1-blocking antibody, but not IL-12- or IL-18-blocking antibodies, significantly inhibited MAIT cell proliferation (Figure 5d), demonstrating that E. coli-induced MAIT cell expansion is largely TCR dependent; this was further confirmed with enriched $\mathrm{CD} 8^{+}$ T cells (Supplementary Figure S5A). Of note, proliferation of MAIT cells at 10BpC could not be completely inhibited by the anti-MR1-blocking antibody, suggesting that other cytokines may be contributing to their proliferation. Indeed, CD161 ${ }^{++}$ $\mathrm{CD}^{+} \mathrm{T}$ cells readily proliferated in response to IL-2, IL-15, or IL-12 in the absence of exogenous TCR stimulation (Supplementary Figure S4D-G).

We next probed how the cytotoxic prolife of MAIT cells changed as they proliferated. PFA-fixed E. coli were added to CTV-stained PBMCs, and after 6 days GrB, perforin, GrK, and GrA expression was assessed. MAIT cells cultured with E. coli acquired a highly cytotoxic profile; $\sim 100 \%$ of cells expressed GrB and $\sim 90 \%$ of cells expressed perforin. GrB and perforin expression did not require proliferation, as $\mathrm{CTV}^{\text {high }}$, nonproliferating cells also expressed high levels of both cytotoxic mediators (Figure 5e). Although resting MAIT cells all express GrA, stimulated MAIT cells upregulated GrA as measured by geometric mean fluorescence intensity (Figure 5f). In turn, there was a significant loss of GrK expression by activated MAIT cells (Figure 5f).

Therefore, MAIT cells are highly proliferative to antigenic and cytokine stimuli in vitro, and antigenic stimulation, irrespective of proliferation, is associated with increased expression of perforin, $\mathrm{GrB}$, and $\mathrm{GrA}$, and decreased expression of GrK.

\section{DISCUSSION}

In this article, we demonstrate that the unique cytotoxic granule content of resting MAIT cells changes markedly upon activation, licensing these cells to kill target cells with greater efficiency. Importantly, to our knowledge this is the first study to investigate the regulation of MAIT cell cytotoxicity and their ability to kill APCs. Our findings support a role for MAIT cells in the control of intracellular bacterial infection.

At rest, almost all CD161 ${ }^{++} \mathrm{CD}^{+} \mathrm{T}$ cells, including MAIT cells, express GrA and GrK within cytotoxic granules and, therefore, are poised to release these immediately upon degranulation. In contrast, they express low levels of perforin, no GrB, and little granulysin or FasL (Supplementary Figure S2E and F). GrA and GrK are closely related trypsin-like serine proteases ${ }^{15}$ originally believed to function as pro-apoptotic proteases, ${ }^{31-33}$ however, recent studies have suggested that they are inefficient inducers of cell death. ${ }^{21,22,34}$ Human GrA is significantly less cytotoxic than mouse $\mathrm{GrA},{ }^{35,36}$ and in virusspecific $\mathrm{CD} 8^{+} \mathrm{T}$ cells, cytotoxicity inversely correlated with GrK expression, whereas GrA expression did not correlate with lytic ability. ${ }^{17}$ Instead, GrA and GrK have been shown to activate monocytes to produce proinflammatory cytokines such as IL-1 $\beta$ and tumor necrosis factor- $\alpha .^{21-23,34}$ Intriguingly, a recent paper by Wensink et al. ${ }^{37}$ has demonstrated in vivo and in vitro that GrK binds to Gram-negative bacteria and lipopolysaccharide, synergistically increasing lipopolysaccharide-induced release of proinflammatory cytokines. GrA release by $\gamma \delta \mathrm{T}$ cells is also critical in the control of BCG growth by inducing tumor necrosis factor- $\alpha$ production in infected macrophages, ${ }^{38}$ and similarly, degranulation of resting MAIT cells may assist in the control of bacterial infection through phagocyte activation. Once GrA and GrK are released from the cell, the signals that may increase the production of these granzymes and aid the replenishment of the granules are unknown. Of note, direct stimulation of MAIT cells with various cytokines such as IL-12 + IL-18 did not significantly increase the amount of GrA or GrK expressed by ex vivo MAIT cells as measured by geometric mean fluorescence intensity, although IL-15 induced a slight increase in GrA in 6/10 donors

(Supplementary Figure S2C and D).

Following antigen exposure, MAIT cells rapidly transition to a more classical cytotoxic cell type, displaying high levels of $\mathrm{GrB}$ and perforin. This is consistent with previous work showing that activated MAIT cells can secrete $\mathrm{GrB},{ }^{8}$ and that $\mathrm{GrB}$ expression in $\mathrm{CD}_{8}{ }^{+} \mathrm{T}$ cells positively correlates with antigenic stimulation. ${ }^{17,39}$ When we probed the mechanisms controlling the early upregulation of GrB by activated MAIT cells, we found this to be dependent upon engagement of the TCR with MR1 and IL-12 or costimulation through CD2/CD28. This is in contrast to degranulation, which is dependent solely on the interaction of the TCR with MR1, and perforin upregulation, which could be induced by multiple cytokines. Among the granzymes, GrB is the most potent, especially in humans where it induces apoptosis in a caspase-independent manner. ${ }^{15,40}$ Thus, the requirement for a second signal for maximal expression of GrB in TCR-stimulated MAIT cells may be important to prevent unwanted tissue damage. The relative importance of MR1- and cytokine-mediated regulation of MAIT cell cytotoxicity in vivo will be crucial in harnessing the cytotoxic potential of these cells. Our data also suggest that the transcription factors Blimp1 and T-bet may be involved in this regulation of GrB expression in MAIT cells. In conventional $\mathrm{CD}^{+} \mathrm{T}$ cells, T-bet and Blimp1 are independently regulated, with Blimp1 deficiency resulting in a failure to produce GrB. ${ }^{25,26}$ Whether Blimp1 and T-bet has a similarly critical role in GrB expression in MAIT cells remains to be determined.

Highly cytotoxic, licensed MAIT cells were able to kill up to $70 \%$ of E. coli-exposed BCLs. Although both ex vivo and licensed MAIT cells degranulated upon exposure to target cells, ex vivo MAIT cells showed significantly less cytotoxicity compared with licensed MAIT cells, consistent with changes in granule content with activation. Blocking experiments showed killing by these licensed MAIT cells was dependent on TCRmediated degranulation, and not Fas-mediated killing, which is important in invariant natural killer T-cell cytotoxicity. ${ }^{41}$

Interestingly, $\mathrm{CD} 161^{++}$cells within the few $\mathrm{CD} 8^{-} \mathrm{T}$ cells in the killing assays degranulated in response to $E$. coli, and indeed $\mathrm{CD} 8{ }^{-} \mathrm{CD} 4^{-}$MAIT cells could be activated by $E$. coli as readily as CD8 ${ }^{+}$MAIT cells (Supplementary Figure S6). Although we cannot rule out $\mathrm{CD} 8$ downregulation, conventional $\mathrm{CD} 8^{+}$ 
T-cell responses also do not necessarily require, but can be augmented by, the CD8 coreceptor. In conventional CD8 ${ }^{+} \mathrm{T}$ cells, CD8 coreceptor enhances the binding of the TCR to the peptide-major histocompatibility complex, and cytolytic responses may be abrogated when coreceptor binding is inhibited. ${ }^{42}$ However, in the context of high-affinity agonist ligands, APCs expressing wild-type or CD8-null human leukocyte antigen molecules induces $\mathrm{CD} 107 \alpha$ externalization by $\mathrm{CD} 8^{+} \mathrm{T}$ cells to a similar extent across a range of peptide concentrations, whereas abrogation of coreceptor binding inhibits degranulation in response to low-affinity peptide variants, even at high peptide concentrations. ${ }^{43}$ Molecular analyses have shown that MR1 can accommodate a range of riboflavin-derived ligands with different binding affinities, ${ }^{10,44}$ and given that murine MAIT cells are mostly CD $8{ }^{-},{ }^{10}$ the role of the CD8 coreceptor in MAIT cell cytotoxicity and biology is a question that warrants further investigation.

The ability of activated MAIT cells to kill target cells may have an important role in bacterial control in vivo. A recent paper showed that MAIT cells can kill epithelial cells infected with $S$. flexneri ${ }^{14}$ suggesting that this function may be important for preventing cytosolic bacteria from crossing the mucosal barrier. Other intracellular bacteria such as Mycobacterium tuberculosis can replicate in macrophages ${ }^{45}$ and B cells, ${ }^{46}$ surviving inside a segregated endosomal compartment. Efficient killing of infected APCs by MAIT cells may lead to enhanced clearance of such bacteria. Intriguingly, an in vivo model using BCG found that IL-12 rather than recognition of MR1 by murine MAIT cells was required for their control of BCG growth, ${ }^{4}$ suggesting that degranulation was not critical in this model. Similarly, murine MAIT cell control of $F$. tularensis growth in vitro was mediated by cytokine production, not infected cell lysis. ${ }^{6}$ Successful killing of infected cells will ultimately depend upon various factors including ligand availability for MR1 loading, inhibitors of cytolysis expressed by the infected cell, ${ }^{47,48}$ and inhibitory/costimulatory signals. Indeed, we found that CD161 is a costimulatory molecule on MAIT cells, and its ligation induces a substantial increase in target cell killing (Fergusson et al., in press, ${ }^{49}$ and Smith et al., unpublished data). Thus, whether cytotoxicity has a significant role in protection mediated by MAIT cells in vivo will most likely depend on both the pathogen and the infected host cell in question.

In this study, we also demonstrate that adult MAIT cells proliferate in response to cytokines as well as TCR stimulation. MAIT cell expansion could be induced through E. coli stimulation in an MR1-dependent manner, a property recently shown to be acquired during fetal development. ${ }^{50}$ Interestingly, MAIT cells downregulated CD161 with increasing rounds of cell division (Supplementary Figure S5B), in agreement with previous observations that MAIT cell clones can be $\mathrm{CD} 161^{-}$. $^{3}$ In our hands, we do not observe CD161 downregulation during short-term activation of MAIT cells (Supplementary Figure S3), as has been shown previously, ${ }^{51}$ but as CD161 costimulates T-cell proliferation, ${ }^{52}$ downregulation of CD161 with proliferation may be beneficial for the continued survival of the cells by limiting activation-induced cell death. In contrast to previous studies, ${ }^{8,53}$ a greater proportion of $\mathrm{CD} 161^{++}$ $\mathrm{CD}^{+} \mathrm{T}$ cells, including the V $\alpha 7.2^{+}$MAIT cells, proliferated when stimulated with IL-2 or IL-15 compared with CD161 ${ }^{+}$ $\mathrm{CD}^{+} \mathrm{T}$ cells, whereas phytohaemagglutinin induced limited proliferation despite high activation (Supplementary Figure S5C and D). Given the sensitivity of MAIT cells to activation-induced cell death, ${ }^{27,54}$ MAIT cell proliferation most likely depends on a balance between expansion and activation-induced cell death, and may explain the variation between MAIT cell proliferation studies. In vivo, once MAIT cells are attracted to sites of infection/ inflammation, their functional impact, including cytotoxic activity, may be further perpetuated by their proliferation.

Overall, our results show that MAIT cell cytotoxicity is suppressed in the resting state, but upon continued exposure to bacteria, they rapidly acquire a cytotoxic phenotype, licensing them to kill target cells. Maximum expression of GrB requires both TCR stimulation and a second signal, provided by IL-12 or costimulation, whereas perforin can be induced by multiple factors. Release of these cytotoxic mediators, however, is ultimately dependent on MR1. This tight regulation of MAIT cell cytotoxicity may have an important role in the control of intracellular bacterial infections, such as M. tuberculosis, while limiting immune-mediated pathology. Further elucidation of the relative role of MR1-dependent and cytokine-mediated regulation of MAIT cell function in vivo will be important in addressing this issue.

\section{METHODS}

Donors. Healthy donors were laboratory volunteers and leukocyte cones (NHS Blood Services, Oxford, UK). Umbilical cord blood samples were provided by the NHS Blood Services. All subjects were recruited in agreement with the local ethics committees of all participating institutions. PBMCs were isolated using Lymphoprep (AxisShield, Oslo, Norway). Cells were thawed and washed in RPMI 1640 with $10 \%$ fetal calf serum, L-glutamine, and penicillin/streptomycin (all Sigma-Aldrich, Dorset, UK).

Flow cytometry. For immunofluorescence staining, dead cells were excluded with the Near-IR Dead-Cell stain (Invitrogen, Paisley, UK). Antibodies used were: anti-CD3 PE-Cy7 or APC, anti-CD8 PerCP-Cy5.5 or eFluor 450, anti-CD69 FITC (eBioscience, Hatfield, UK); anti-CD161 PE or APC, anti-CD4 VioGreen (Miltenyi Biotec, Surrey, UK); anti-V $\alpha 7.2$ PE or FITC or PE-Cy7, anti-CD107 $\alpha$ PE-Cy7, anti-GrA PerCP-Cy5.5, anti-Perforin Pacific Blue, anti-granulysin PE, anti-FasL PE (Biolegend, London, UK); anti-GrB AlexaFluor700, antiPerforin FITC, anti-Ki67 FITC (BD Biosciences, Oxford, UK), antiGrB APC (Invitrogen); anti-GrK FITC (Immunotools, Friesoythe, Germany); anti-T-bet PE (Santa Cruz Biotechnology, Heidelberg, Germany); anti-GrA FITC, anti-Blimp1 AlexaFluor488 (R\&D Systems, Abingdon, UK); and anti-CD $8 \beta$ PE (Beckman Coulter, High Wycombe, UK).

Data were collected on the flow cytometers LSRII (BD Biosciences) or MACSQuant (Miltenyi Biotec), and was analyzed using FlowJo v9.6 (TreeStar, Ashland, OR). For ImageStream analysis, see Supplementary Methods.

In vitro stimulation. E. coli (DH5 $\alpha$, Invitrogen) was fixed in $2 \%$ PFA for $20 \mathrm{~min}$, and washed extensively. A sterility control was prepared in identical manner and used as a negative control. In coculture experiments, E. coli, or the negative control, was added to THP1 cells (ECACC, Wiltshire, UK) at 25 Bacteria-per-Cell (BpC) overnight. Subsequently, THP1 cells were washed and $\mathrm{CD} 8^{+} \mathrm{T}$ cells isolated 
using CD8 Microbeads (Miltenyi Biotec; purities were $\geq 90 \%$ ) and anti-CD107 $\alpha$ antibody were added to the wells overnight. For further details, see Supplementary Methods.

Killing assays. The assay was based on the FATAL assay. ${ }^{28}$ BCLs were either coincubated with PFA-fixed E. coli at $25 \mathrm{BpC}$ or the negative control overnight. Cells were harvested and the E. coli-exposed BCLs were stained with CellTrace CFSE (CFSE; Invitrogen), whereas the negative control BCLs were stained with CTV (Invitrogen) according to the manufacturer's protocol. After extensive washing, the CFSEand CTV-labeled cells were mixed at a 1:1 ratio before plating.

$\mathrm{CD}^{+}{ }^{+} \mathrm{T}$ cells were isolated from PBMCs using CD8 Microbeads (Miltenyi Biotec), and added to the wells at the indicated E:T ratios overnight, with an anti-CD107 $\alpha$ antibody. E:T indicates ratio of CD ${ }^{+}$ T cells to $\mathrm{CFSE}^{+}$target cells, unless otherwise indicated. CD8 ${ }^{+} \mathrm{T}$-cell purities were $\geq 90 \%$. Experiments were done in duplicate. An MR1blocking antibody (clone 26.5), a FasL-blocking antibody (clone NOK1 , BioLegend) or an appropriate isotype control was added at $5 \mu \mathrm{g} \mathrm{ml}^{-1}$ for blocking experiments. To block degranulation, $5 \mathrm{~mm}$ ethylene glycol tetra-acetic acid (Sigma-Aldrich) with $1 \mathrm{~mm} \mathrm{MgCl}_{2}$ (Affymetrix, High Wycombe, UK) was used.

Following incubation, cells were stained with a live-dead maker (Invitrogen) before fixation/permeabilization for the staining of relevant markers. Gating is shown in Supplementary Figure S4A. Based on the \%survival of $\mathrm{CFSE}^{+}$cells in wells containing no effectors $(\mathrm{E}: \mathrm{T}=0: 1)$, the expected frequency of target cells can be found:

$$
\text { Expected ratio }(\mathrm{ER})=\% \mathrm{CFSE}^{+} \text {cells } / \% \mathrm{CTV}^{+} \text {cells }
$$

Using this ratio, the \% specific killing can be calculated:

$$
\begin{aligned}
\% \text { specific killing }= & 100 \times\left[\left(\mathrm{ER} \times \% \mathrm{CTV}^{+} \text {cells }\right)-\% \mathrm{CFSE}^{+} \text {cells }\right] / \\
& \left(\mathrm{ER} \times \% \mathrm{CTV}^{+} \text {cells }\right)
\end{aligned}
$$

For the post hoc estimation of the actual E:T ratio in each well, the frequency of $\mathrm{CD} 161^{++} \mathrm{CD} 8{ }^{+} \mathrm{T}$ cells within live cells was divided by the expected frequency of CFSE ${ }^{+}$cells in each well $\left(\mathrm{ER} \times \% \mathrm{CTV}^{+}\right.$ cells).

Proliferation assays. PBMCs were labeled with CTV (Invitrogen) according to the manufacturer's protocol, and cultured for 6 days with either PFA-fixed E. coli at the indicated BpC, or the sterility control. An MR1-blocking antibody (clone 26.5), IL-12p40/70-blocking antibody (eBioscience), IL-18-blocking antibody (clone 126-2H, MBL International, Woburn, MA) or an appropriate isotype control was used at $5 \mu \mathrm{g} \mathrm{ml}^{-1}$ for blocking experiments. Alternatively, THP1 cells were cultured with $E$. coli at $25 \mathrm{BpC}$ overnight before irradiation and were cocultured with CTV-labeled $\mathrm{CD}^{+}{ }^{+} \mathrm{T}$ cells enriched with CD8 Microbeads for 6 days, with autologous irradiated feeder cells. For cytokine-induced proliferation, CTV-labeled PBMCs were cultured for 6 days with the following stimuli: recombinant human IL-2 $\left(100 \mathrm{IU} \mathrm{ml}^{-1}\right), \mathrm{IL}-15\left(25 \mathrm{ng} \mathrm{ml}^{-1}\right)$, IL-18 $\left(50 \mathrm{ng} \mathrm{ml}^{-1}\right)$, IL-12 $\left(50 \mathrm{ng} \mathrm{ml}^{-1}\right)$, phytohaemagglutinin $\left(2 \mu \mathrm{g} \mathrm{ml}^{-1}\right)$, anti-CD3-coated or anti-CD3/ CD28/CD2-coated beads (Miltenyi Biotec) at a 1:1 ratio, or the indicated combinations.

Statistical analysis. All graphs and statistical analyses were completed using GraphPad Prism software Version 6.0b (La Jolla, CA). Coexpression analysis was performed with SPICE software Version 5.3. ${ }^{55}$ Statistical significance was assessed using paired Student's $t$-test, repeated-measures one-way analysis of variances or two-way analysis of variances, with Bonferroni's correction for multiple comparison assays. All data presented as means with s.e.m., unless otherwise indicated.

SUPPLEMENTARY MATERIAL is linked to the online version of the paper at http://www.nature.com/mi

\section{ACKNOWLEDGMENTS}

This work was supported by the Wellcome Trust (WT091663MA); the Medical Research Council; the NIHR Biomedical Research Centre (Oxford); the Nuffield Department of Clinical Medicine (Oxford); the James Martin School for the 21st Century (Oxford); the National Institutes of Health (NIAD 5U19A082630 and Al046553), and the Oxford Dominions Trust.

\section{DISCLOSURE}

The authors declared no conflict of interest.

c 2015 Society for Mucosal Immunology

\section{REFERENCES}

1. Treiner, E. et al. Selection of evolutionarily conserved mucosal-associated invariant T cells by MR1. Nature 422, 164-169 (2003).

2. Le Bourhis, L. et al. Antimicrobial activity of mucosal-associated invariant T cells. Nat. Immunol. 11, 701-708 (2010).

3. Gold, M.C. et al. Human mucosal associated invariant T cells detect bacterially infected cells. PLoS Biol. 8, e1000407 (2010).

4. Chua, W.J. etal. Polyclonal mucosal-associated invariant Tcells have unique innate functions in bacterial infection. Infect. Immun. 80, 3256-3267 (2012).

5. Georgel, P., Radosavljevic, M., Macquin, C. \& Bahram, S. The nonconventional MHC class I MR1 molecule controls infection by Klebsiella pneumoniae in mice. Mol. Immunol. 48, 769-775 (2011).

6. Meierovics, A., Yankelevich, W.-J.C. \& Cowley, S.C. MAIT cells are critical for optimal mucosal immune responses during in vivo pulmonary bacterial infection. Pro. Natl. Acad. Sci. USA 110, E3119-E3128 (2013).

7. Kjer-Nielsen, L. etal. MR1 presents microbial vitamin B metabolites to MAIT cells. Nature 491, 717-723 (2012).

8. Dusseaux, M. et al. Human MAIT cells are xenobiotic-resistant, tissuetargeted, CD161 hi IL-17-secreting T cells. Blood 117, 1250-1259 (2011).

9. Tang, X.-Z. et al. IL-7 licenses activation of human liver intrasinusoidal mucosal-associated invariant Tcells. J. Immunol. 190, 3142-3152 (2013).

10. Reantragoon, R. et al. Antigen-loaded MR1 tetramers define Tcell receptor heterogeneity in mucosal-associated invariant T cells. J. Ex. Med 210, 2305-2320 (2013).

11. Walker, L.J. et al. Human MAIT and CD $8 \alpha \alpha$ cells develop from a pool of type-17 precommitted CD8 + T cells. Blood 119, 422-433 (2012).

12. Billerbeck, E. et al. Analysis of CD161 expression on human CD8 + Tcells defines a distinct functional subset with tissue-homing properties. Proc. Natl. Acad. Sci. USA 107, 3006-3011 (2010).

13. Ussher, J.E. et al. CD $161^{++} \mathrm{CD} 8^{+}$T cells, including the MAIT cell subset, are specifically activated by IL-12 + IL-18 in a TCR-independent manner. Eur. J. Immunol. 44, 195-203 (2014).

14. LeBourhis, L. et al. MAITcells detect and efficiently lyse bacterially-infected epithelial cells. PLoS Pathog. 9, e1003681 (2013).

15. Chowdhury, D. \& Lieberman, J. Death by athousand cuts: granzyme pathways of programmed cell death. Annu. Rev. Immunol. 26, 389-420 (2008).

16. Pinkoski, M.J. etal. Entry and trafficking of granzyme B in target cells during granzyme B-perforin-mediated apoptosis. Blood 92, 1044-1054 (1998).

17. Harari, A., Enders, F.B., Cellerai, C., Bart, P.A. \& Pantaleo, G. Distinct profiles of cytotoxic granules in memory CD8 T Cells correlate with function, differentiation stage, and antigen exposure. J. Virol. 83, 2862-2871 (2009).

18. Wolint, P., Betts, M.R., Koup, R.A. \& Oxenius, A. Immediate cytotoxicity but not degranulation distinguishes effector and memory subsets of CD8 $+\mathrm{T}$ cells. J. Exp. Med. 199, 925-936 (2004).

19. Bratke, K., Kuepper, M., Bade, B., Virchow, J.C. \& Luttmann, W. Differential expression of human granzymes $\mathrm{A}, \mathrm{B}$, and $\mathrm{K}$ in natural killer cells and during CD8 $+\mathrm{T}$ cell differentiation in peripheral blood. Eur. J. Immunol. 35, 2608-2616 (2005)

20. Curtsinger, J.M., Lins, D.C., Johnson, C.M. \& Mescher, M.F. Signal 3 tolerant CD8 T cells degranulate in response to antigen but lack granzyme B to mediate cytolysis. J. Immunol. 175, 4392-4399 (2005).

21. Metkar, S.S. et al. Human and mouse granzyme A induce a proinflammatory cytokine response. Immunity 29, 720-733 (2008).

22. Cooper, D.M., Pechkovsky, D.V., Hackett, T.L., Knight, D.A. \& Granville, D.J. Granzyme K activates protease-activated receptor-1. PLoS One 6, e21484 (2011). 
23. Sower, L.E. et al. Extracellular activities of human granzyme A. Monocyte activation by granzyme A versus alpha-thrombin. J. Immunol. 156, 2585-2590 (1996).

24. Makedonas, G. et al. Rapid up-regulation and granule-independent transport of perforin to the immunological synapse define a novel mechanism of antigen-specific CD8 + Tcell cytotoxic activity. J. Immunol. 182, 5560-5569 (2009).

25. Kallies, A., Xin, A., Belz, G.T. \& Nutt, S.L. Blimp-1 transcription factor is required for the differentiation of effector CD8 $+\mathrm{T}$ Cells and memory responses. Immunity 31, 283-295 (2009).

26. Cruz-Guilloty, F. et al. Runx3 and T-box proteins cooperate to establish the transcriptional program of effector CTLs. J. Exp. Med. 206, 51-59 (2009).

27. Cosgrove, C. et al. Early and nonreversible decrease of CD161 ${ }^{++}$/MAIT cells in HIV infection. Blood 121, 951-961 (2013).

28. Sheehy, M.E., McDermott, A.B., Furlan, S.N., Klenerman, P. \& Nixon, D.F. A novel technique for the fluorometric assessment of $\mathrm{T}$ lymphocyte antigen specific lysis. J. Immunol. Methods 249, 99-110 (2001).

29. Betts, M.R. et al. Sensitive and viable identification of antigen-specific $\mathrm{CD} 8+\mathrm{T}$ cells by a flow cytometric assay for degranulation. J. Immunol. Methods 281, 65-78 (2003).

30. Poupot, M., Fournié, J.-J. \& Poupot, R. Trogocytosis and killing of IL-4polarized monocytes by autologous NK cells. J. Leukoc. Biol. 84, 1298-1305 (2008).

31. Jiang, W., Chai, N.R., Maric, D. \& Bielekova, B. Unexpected role for granzyme K in CD56 ${ }^{\text {bright }} \mathrm{NK}$ cell-mediated immunoregulation of multiple sclerosis. J. Immunol. 187, 781-790 (2011).

32. Jenkins, M.R., Trapani, J.A., Doherty, P.C. \& Turner, S.J. Granzyme K expressing cytotoxic $T$ lymphocytes protects against influenza virus in granzyme $\mathrm{AB}^{-1-}$ Mice. Viral Immunol. 21, 341-346 (2008).

33. Zhao, T. et al. Granzyme K cleaves the nucleosome assembly protein SET to induce single-stranded DNA nicks of target cells. Cell Death Differ. 14, 489-499 (2007)

34. Joeckel, L.T. et al. Mouse granzyme K has pro-inflammatory potential. Cell Death Differ. 18, 1112-1119 (2011).

35. Kaiserman, D. et al. Identification of Serpinb6b as a speciesspecific mouse granzyme A inhibitor suggests functional divergence between human and mouse granzyme A. J. Biol. Chem. 289, 9408-9417 (2014).

36. Susanto, O. et al. Mouse granzyme A induces a novel death with writhing morphology that is mechanistically distinct from granzyme B-induced apoptosis. Cell Death Differ. 20, 1183-1193 (2013).

37. Wensink, A.C. et al. Granzyme K synergistically potentiates LPS-induced cytokine responses in human monocytes. Proc. Natl. Acad. Sci. USA 111, 5974-5979 (2014)

38. Spencer, C.T. et al. Granzyme A produced by $\gamma_{9} \delta_{2} T$ cells induces human macrophages to inhibit growth of an intracellular pathogen. PLoS Pathog. 9, e1003119 (2013).

39. Migueles, S.A. et al. Lytic granule loading of CD8 + T cells is required for HIV-infected cell elimination associated with immune control. Immunity 29 1009-1021 (2008).
40. Kaiserman, D. et al. The major human and mouse granzymes are structurally and functionally divergent. J. Cell Biol. 175, 619-630 (2006).

41. Wingender, G., Krebs, P., Beutler, B. \& Kronenberg, M. Antigen-specific cytotoxicity by invariant NKT cells in vivo is CD95/CD178-dependent and is correlated with antigenic potency. J. Immunol. 185, 2721-2729 (2010).

42. Purbhoo, M.A. et al. The human CD8 coreceptor effects cytotoxic T cell activation and antigen sensitivity primarily by mediating complete phosphorylation of the Tcell receptor chain $\zeta$. J. Biol. Chem. 276, 32786-32792 (2001).

43. Laugel, B. et al. Different $T$ cell receptor affinity thresholds and CD8 coreceptor dependence govern cytotoxic T lymphocyte activation and tetramer binding properties. J. Biol. Chem. 282, 23799-23810 (2007).

44. López-Sagaseta, J. et al. MAIT recognition of a stimulatory bacterial antigen bound to MR1. J. Immunol. 191, 5268-5277 (2013).

45. Buckner, M.M.C. \& Finlay, B.B. Host-microbe interaction: innate immunity cues virulence. Nature 472, 179-180 (2011).

46. Souwer, Y. et al. Selective infection of antigen-specific B lymphocytes by Salmonella mediates bacterial survival and systemic spreading of infection. PLoS One 7, e50667 (2012).

47. Toossi, Z. et al. Induction of serine protease inhibitor 9 by Mycobacterium tuberculosis inhibits apoptosis and promotes survival of infected macrophages. J. Infect. Dis. 205, 144-151 (2012).

48. Willberg, C.B. et al. Protection of hepatocytes from cytotoxic T cell mediated killing by interferon-alpha. PLoS One 2, e791 (2007).

49. Fergusson, R.J. et al. CD161 defines a transcriptional and functional phenotype shared across distinct human Tcell lineages. Cell. Rep. (in press).

50. Leeansyah, E., Loh, L., Nixon, D.F. \& Sandberg, J.K. Acquisition of innatelike microbial reactivity in mucosal tissues during human fetal MAIT-cell development. Nat. Commun 5, 3143 (2014).

51. Leeansyah, E. et al. Activation, exhaustion, and persistent decline of the antimicrobial MR1-restricted MAIT-cell population in chronic HIV-1 infection. Blood 121, 1124-1135 (2013).

52. Germain, C. et al. Induction of lectin-like transcript 1 (LLT1) protein cell surface expression by pathogens and interferon- $\gamma$ contributes to modulate immune responses. J. Biol. Chem. 286, 37964-37975 (2011).

53. Turtle, C.J. et al. Innate signals overcome acquired TCR signaling pathway regulation and govern the fate of human $\mathrm{CD} 161^{\mathrm{hi}} \mathrm{CD} 8 \alpha^{+}$semi-invariant T cells. Blood 118, 2752-2762 (2011).

54. Gérart, S. et al. Human iNKT and MAIT cells exhibit a PLZF-dependent proapoptotic propensity that is counterbalanced by XIAP. Blood 121, 614-623 (2013).

55. Roederer, M., Nozzi, J.L. \& Nason, M.C. SPICE: exploration and analysis of post-cytometric complex multivariate datasets. Cytometry A 79, 167-174 (2011)

(1) (3) This work is licensed under the Creative Commons Attribution-NonCommercial-No Derivative Works 3.0 Unported License. To view a copy of this license, visit http://creativecommons.org/licenses/by-nc-nd/3.0/ 